\title{
Planar, strong magnetic field source for a chip ion trap
}

Cite as: Rev. Sci. Instrum. 91, 103201 (2020); https://doi.org/10.1063/5.0024735

Submitted: 11 August 2020 . Accepted: 30 September 2020 . Published Online: 14 October 2020

(iD) Jonathan Pinder, (D) John H. Lacy, (D) Ryan Willetts, (i) April Cridland Mathad, (D) Alberto Uribe, and (D) José Verdú

\section{ARTICLES YOU MAY BE INTERESTED IN}

Coherent coupling of a trapped electron to a distant superconducting microwave cavity Applied Physics Letters 117, 154001 (2020); https://doi.org/10.1063/5.0023002

A cryogenic setup for multifunctional characterization of luminescence and scintillation properties of single crystals

Review of Scientific Instruments 91, 103108 (2020); https://doi.org/10.1063/5.0016175

Picosecond solid-state generator with a peak power of $50 \mathrm{GW}$

Review of Scientific Instruments 91, 104705 (2020); https://doi.org/10.1063/5.0017980

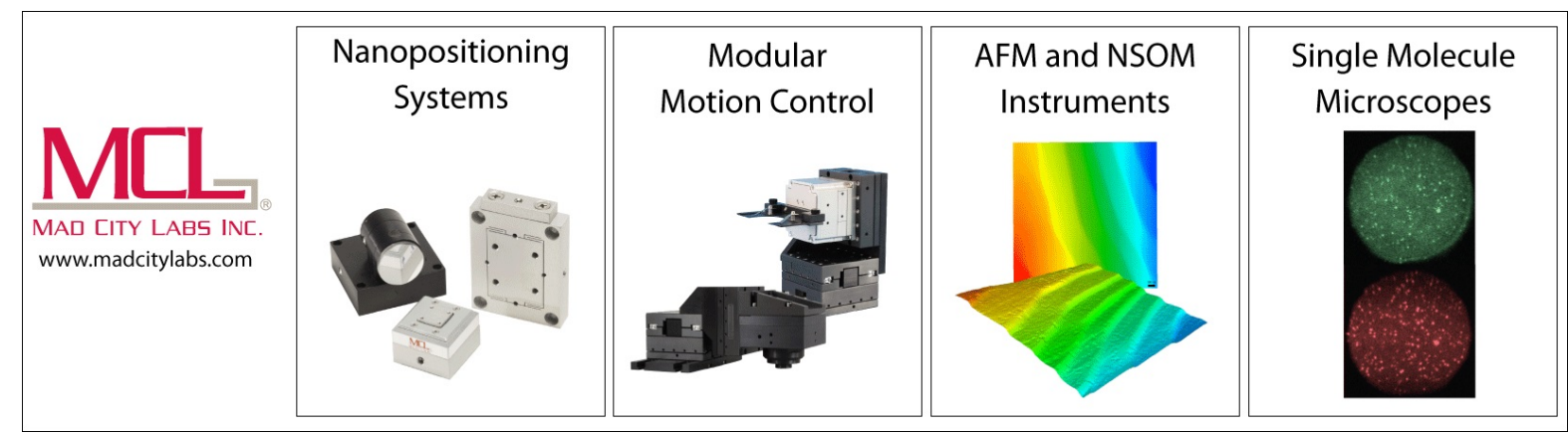




\title{
Planar, strong magnetic field source for a chip ion trap
}

\author{
Cite as: Rev. Sci. Instrum. 91, 103201 (2020); doi: 10.1063/5.0024735 \\ Submitted: 11 August 2020 - Accepted: 30 September 2020 • \\ Published Online: 14 October 2020

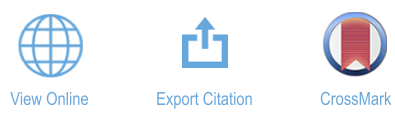

\begin{abstract}
Jonathan Pinder, ${ }^{1}$ (D) John H. Lacy, ${ }^{2}$ (D) Ryan Willetts,' (D) April Cridland Mathad, ${ }^{3}$ (D) Alberto Uribe, and José Verdú ${ }^{1, a}$
\end{abstract}

\author{
AFFILIATIONS \\ ${ }^{1}$ Department of Physics and Astronomy, University of Sussex, Falmer BN1 9QH, United Kingdom \\ ${ }^{2}$ Physics Department, Williams College, Williamstown, Massachusetts 01267, USA \\ ${ }^{3}$ Physics Department, Swansea University, Swansea SA2 8PP, United Kingdom
}

a) Author to whom correspondence should be addressed: jlv20@sussex.ac.uk

\begin{abstract}
We present a planar, scalable magnetic field source, originally conceived for a chip ion trap. It consists of two symmetric sections, each with several independent currents arranged in coplanar, concentric rectangular loops. The currents allow for tuning the strength of the field and its lowest-order derivatives at one discretional position along the source's vertical symmetry axis, a few $\mathrm{mm}$ above its surface. We describe the construction and calibration of the device and the cryogenic setup. The two most important current configurations for a Penning ion trap, the homogeneous field and the magnetic bottle, are investigated experimentally. Homogeneous fields around $0.5 \mathrm{~T}$ are routinely reached. We discuss the maximum attainable field, and we briefly describe ongoing further developments aiming at homogeneous fields well above $1 \mathrm{~T}$.
\end{abstract}

Published under license by AIP Publishing. https://doi.org/10.1063/5.0024735

\section{INTRODUCTION}

Superconducting solenoids are used in a wide variety of applications where a static and homogeneous magnetic field in the Tesla range is required, such as magnetic resonance imaging (MRI), quantum optics with nuclear magnetic resonance (NMR) systems, Fourier-Transform Ion-Cyclotron Resonance (FT-ICR) mass spectrometry, ${ }^{3-5}$ Penning traps for precision measurements in atomic and nuclear physics, ${ }^{6}$ and others. The solenoids can provide a region of the order of a few $\mathrm{cm}^{3}$ where the magnetic field has essentially only one component, commonly denoted by $B_{z}\left(\hat{u}_{z}=\right.$ solenoid's symmetry axis), and whose strength is constant within a few parts per million (ppm) or better. Time stabilities as high as $\sim 20 \mathrm{ppt} / \mathrm{h}$ have been demonstrated ${ }^{7}$ using a combination of passive flux-stabilizing coils, ${ }^{8}$ temperature + helium pressure regulation, and active field control. Solenoids made with high temperature superconductors (HTS), such as YBCO and $\mathrm{MgB}_{2}$, are being investigated. ${ }^{9}$ However, most magnets in operation are fabricated with low temperature superconductors (LTS), such as $\mathrm{NbTi}$ and $\mathrm{Nb}_{3} \mathrm{Sn}$. Mainly, this is due to the poor joints of HTS wires achieved so far, which impede persistent mode operation. ${ }^{10}$ Flux pumping can be, in principle, used; ${ }^{11,12}$ however, the magnets are usually energized with sources of currents of many tens of amperes. This requires conducting copper rods of several $\mathrm{cm}^{2}$ cross section and correspondingly bulky thermalization fittings. In overall, the $\mathrm{cm}^{3}$ region of homogeneous field has a footprint cost of $\geq 1 \mathrm{~m}^{3}$ for the solenoid, including its hosting cryostat.

Motivated by the applications of trapped electrons in quantum technology ${ }^{13,14}$ and by the potential miniaturization of FT-ICR mass spectrometry, we have developed a planar Penning trap manufactured in a chip, which we denote geonium chip. ${ }^{15,16}$ The trap's electrodes are assembled on the chip's metallic surface, where -in its current version- they $\operatorname{span}^{17} 1.3 \times 1.5 \mathrm{~cm}^{2}$. Charged particles can be trapped at one selectable height $y_{0}$ up to $\sim 2 \mathrm{~mm}$ above the chip's surface. ${ }^{16}$ Furthermore, unlike conventional FT-ICR spectrometers, where the ion cell is at room temperature, ${ }^{18}$ the geonium chip serves as an ion trap in a cryogenic $4 \mathrm{~K}$ environment. ${ }^{17}$ At that temperature and in a magnetic field of $1 \mathrm{~T}$, the motion of a trapped electron occupies a microscopic volume ${ }^{15}$ of $2 \times 2 \times 70 \mu \mathrm{m}^{3}\left(\hat{u}_{x}, \hat{u}_{y}, \hat{u}_{z}\right)$. A singly charged ion of $200 \mathrm{Da}$ (i.e., 370000 times heavier than the electron) also occupies a small region of $110 \times 110 \times 70 \mu \mathrm{m}^{3}$. Hence, many applications with a single or a few ions/electrons in a cryogenic trap 
do not require a "large" region of a homogeneous magnetic field, spanning several $\mathrm{cm}^{3}$. A smaller volume of only one or a few $\mathrm{mm}^{3}$ would, in principle, suffice. Moreover, if the trapped ion is located at only a few $\mathrm{mm}$ distance from the source, then any given magnetic field strength can be generated with substantially less superconducting material than required in a standard solenoid, where the bore diameter is typically $10 \mathrm{~cm}$ or larger. ${ }^{19}$ Furthermore, the cryostat used for the ion trap can also cool the magnetic field source. Temperature fluctuations in the cold-head of a conventional pulse-tube cryocooler can be reduced below $\pm 10 \mathrm{mK},{ }^{20}$ hence, providing very good thermal stability of the magnetic field source as well. Following these arguments, a planar, scalable magnetic field source-originally for the geonium chip but also useful for other planar traps ${ }^{21}$ - has been proposed and theoretically described. ${ }^{22,23}$ Here, we present its first basic experimental realization and characterization.

\section{THEORETICAL BACKGROUND}

The devised magnetic field source is sketched in Fig. 1. It consists of two symmetric sections, each with four coplanar and concentric rectangular closed loops of current. The closed loop topology allows for persistent current operation. We denote the four independent currents $\left(I_{0}, I_{1}, I_{2}, I_{3}\right)$. For every single $n$-th loop in one section, its current $I_{n}$ runs in opposite sense-clockwise/anticlockwise or vice versa-to that the same current in the mirror-symmetric loop of the other section. Hence, with this configuration, for any position located along the vertical $\hat{u}_{y}$ axis, $\left(0, y_{0}, 0\right)$, the resulting magnetic field is purely "axial," that is, oriented along $\hat{u}_{z} \rightarrow \vec{B}\left(0, y_{0}, 0\right)$ $=B_{z}\left(0, y_{0}, 0\right) \hat{u}_{z}, \forall y_{0}$.

An example of the magnetic field distribution created by the source introduced in Fig. 1 has been computed in Fig. 2. The example illustrates that by choosing adequate currents $\left(I_{0}, I_{1}, I_{2}, I_{3}\right)$, the magnetic field inhomogeneities can be "compensated," i.e., $B_{z}$ becomes constant within some volume around the selected position $\left(0, y_{0}, 0\right)$, and the radial components $B_{y}$ (and $B_{x}$, not shown in the figure) vanish. The detailed mathematical description of such "compensation" 22,23 is summarized now here in Secs. II A-II B 2.

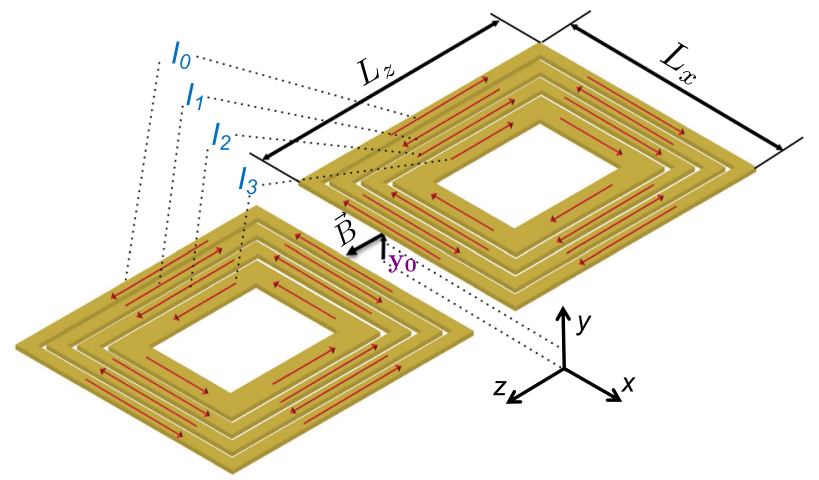

FIG. 1. Model of the planar magnetic field source. It comprises two symmetric sections with four independent pairs of currents. For all four pairs, the one current and its mirror partner run in opposite senses. The currents $\left(I_{0}, I_{1}, I_{2}, I_{3}\right)$ can be tuned to deliver a specific target magnetic field at one position $\left(0, y_{0}, 0\right)$, above the source's surface.

\section{A. Symmetries of the magnetic vector potential}

The magnetic vector potential $\vec{A}(\vec{r})$ (with $\vec{r}=(x, y, z)$ ) for Fig. 1 has only two components: $\vec{A}(\vec{r})=A_{x}(\vec{r}) \hat{u}_{x}+A_{z}(\vec{r}) \hat{u}_{z}$. The missing one, $A_{y} \hat{u}_{y}$, vanishes due to the absence of currents propagating along the vertical $\hat{u}_{y}$ axis. The symmetries of $A_{x}(\vec{r})$ and $A_{z}(\vec{r})$ can be directly inferred from the several mirror (anti)symmetries of the set of current paths parallel to $x$ and $z$ in Fig. 1, respectively. We have

$$
\begin{aligned}
& A_{x}(\vec{r})=A_{x}(-x, y, z) \wedge A_{x}(\vec{r})=A_{x}(x, y,-z), \\
& A_{z}(\vec{r})=-A_{z}(-x, y, z) \wedge A_{z}(\vec{r})=-A_{z}(x, y,-z) .
\end{aligned}
$$

Equations (1) and (2) manifest that $A_{x}(\vec{r})$ is an even function of $x$ and $z$, while $A_{z}(\vec{r})$ is an odd function along those same axes. From the latter, we have $A_{z}(0, y, z)=0 \forall(y, z)$ and $A_{z}(x, y, 0)=0$ $\forall(x, y)$. Hence, we can make the approximation that within a small volume around $\left(0, y_{0}, 0\right)$, the axial component of the vector potential can be neglected: $A_{z} \simeq 0 \Rightarrow \vec{A}(\vec{r}) \simeq A_{x}(\vec{r}) \hat{u}_{x}$. Moreover, the even symmetry of $A_{x}$ implies that all its odd derivatives vanish. In particular, we have $\frac{\partial A_{x}}{\partial x}=0$. Thus, we make the second approximation that again within the same small volume centered on $\left(0, y_{0}, 0\right)$, the function $A_{x}$ is invariant under $x \Rightarrow A_{x} \neq A_{x}(x) \Rightarrow A_{x}=A_{x}(y, z)$. These two approximations can be summarized in one equation: $\vec{A}(\vec{r}) \simeq A_{x}(y, z) \hat{u}_{x}$. This latter expression becomes exact if the currents parallel to $\hat{u}_{x}$ in Fig. 1 are infinitely long: $L_{x} \rightarrow \infty$. For this reason, we refer to it as the "long $L_{x}$ " approximation. In practice, when $y_{0} \ll L_{x}$, for $L_{x}$ being a finite number, it can be shown numerically that this approximation is valid to a few percent or much lower deviation, depending on the actual source dimensions. ${ }^{22,23}$ In Sec. II B 2, we will take those deviations into account in order to explicitly eliminate their effect when compensating the magnetic field.

\section{1. $\vec{A}(\vec{r})$ series expansion in the "long $L_{x}$ " approximation}

Since at the selected position $\left(0, y_{0}, 0\right)$ no currents flow (besides the trapped particle itself), we have $\nabla \times \vec{B}=\overrightarrow{0}$. Moreover, $\nabla \cdot \vec{B}=$ 0 and $\vec{B}=\nabla \times \vec{A}$. Using these and the "long $L_{x}$ " approximation, $\vec{A}(\vec{r}) \simeq A_{x}(y, z) \hat{u}_{x}$, it can be shown ${ }^{22,23}$ that the three-dimensional series expansion of the vector potential around $\left(0, y_{0}, 0\right), \vec{A}=\vec{A}_{0}+$ $\vec{A}_{1}+\vec{A}_{2}+\vec{A}_{3}+\cdots$ is given by the following terms: ${ }^{22,23}$

$$
\begin{gathered}
\vec{A}_{0}=-B_{z, 0}\left(y-y_{0}\right) \hat{u}_{x}, \\
\vec{A}_{1}=\frac{1}{2 !} \frac{\partial B_{z}}{\partial y}\left(z^{2}-\left(y-y_{0}\right)^{2}\right) \hat{u}_{x}, \\
\vec{A}_{2}=\frac{1}{3 !} \frac{\partial^{2} B_{z}}{\partial y^{2}}\left(y-y_{0}\right)\left(3 z^{2}-\left(y-y_{0}\right)^{2}\right) \hat{u}_{x}, \\
\vec{A}_{3}=\frac{1}{4 !} \frac{\partial^{3} B_{z}}{\partial y^{3}}\left(6\left(y-y_{0}\right)^{2} z^{2}-\left(y-y_{0}\right)^{4}-z^{4}\right) \hat{u}_{x} .
\end{gathered}
$$

In Eqs. (3)-(6), the $B_{z}$ derivatives are evaluated at $\left(0, y_{0}, 0\right)$. In general, we define the magnetic $i j k$ - inhomogeneities as

$$
B_{z, i j k}=\left.\frac{1}{i ! j ! k !} \frac{\partial B_{z}}{\partial x^{i} \partial y^{j} \partial z^{k}}\right|_{\left(x=0, y=y_{0}, z=0\right)} ; i, j, k \in \mathbb{N} .
$$

The series expansion terms given in Eqs. (3)-(6) show that within a small region around $\left(0, y_{0}, 0\right)$ where the "long $L_{x}$ " approximation holds, the magnetic field is determined by $\left(B_{z, 0}, B_{z, 010}, B_{z, 020}\right.$, $\left.B_{z, 030}, \ldots\right)$. Therefore, for the source of Fig. 1, in principle, creating 
a) $B_{z}$ (Gauss)

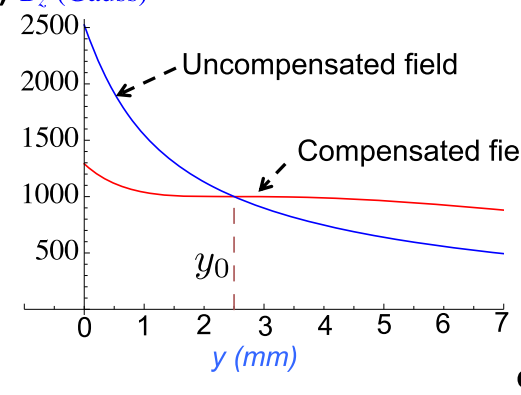

b)

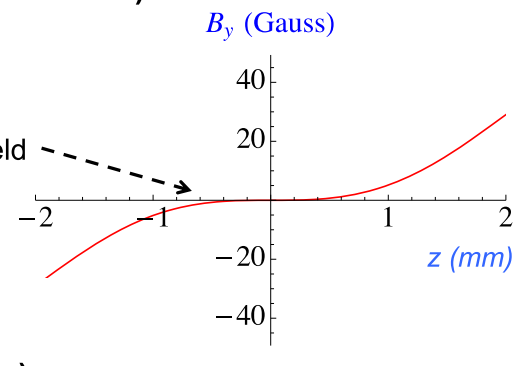

c)

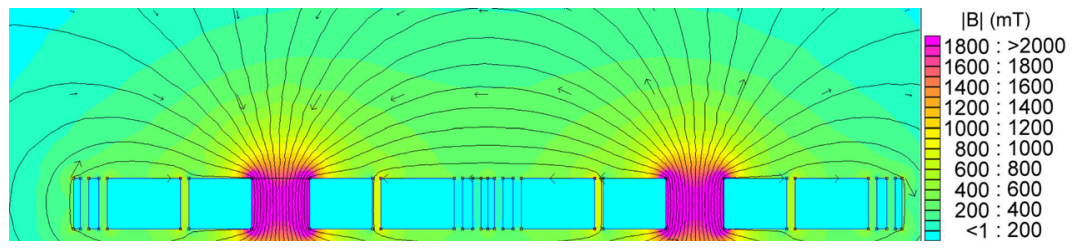

FIG. 2. (a) Plot of the $B_{z}$ magnetic field component along the vertical axis $\hat{u}_{y}$. (b) Plot of the $B_{y}$ component along the axial direction, $\hat{u}_{z}$. (c) Numeric contour plot of the field in the $z-y$ plane, including the Meissner effect. All examples are computed with the source dimensions given in Sec. III A. a homogeneous magnetic field requires canceling only the inhomogeneities of the axial component $B_{z}$, merely along the vertical axis $\hat{u}_{y} \rightarrow B_{z, 010}=B_{z, 020}=B_{z, 030}=\cdots=0$. This results in $\vec{A}_{1}=\vec{A}_{2}=\vec{A}_{3}=$ $\cdots=\overrightarrow{0}$, generating an homogeneous field, $\vec{B}=B_{z, 0} \hat{\mathcal{u}}_{z}$, in the vicinity of $\left(0, y_{0}, 0\right)$.

\section{B. The magnetic field calibration matrix $\Gamma$}

The applied currents $\left(I_{0}, I_{1}, I_{2}, I_{3}\right)$ and the magnetic coefficients $\left(B_{z, 0}, B_{z, 010}, B_{z, 020}, B_{z, 030}\right)$ are univocally linked by a system of linear equations, ${ }^{22,23}$

$$
\left(\begin{array}{c}
B_{z, 0} \\
B_{z, 010} \\
B_{z, 020} \\
B_{z, 030}
\end{array}\right)=\Gamma\left(y_{0}\right) \cdot\left(\begin{array}{c}
I_{0} \\
I_{1} \\
I_{2} \\
I_{3}
\end{array}\right),
$$

where $\Gamma$ is a $4 \times 4$ square matrix, given by

$$
\Gamma\left(y_{0}\right)=\left(\begin{array}{cccc}
b_{z, 0}^{0} & b_{z, 0}^{1} & b_{z, 0}^{2} & b_{z, 0}^{3} \\
b_{z, 010}^{0} & b_{z, 010}^{1} & b_{z, 010}^{2} & b_{z, 010}^{3} \\
b_{z, 020}^{0} & b_{z, 020}^{1} & b_{z, 020}^{2} & b_{z, 020}^{3} \\
b_{z, 030}^{0} & b_{z, 030}^{1} & b_{z, 030}^{2} & b_{z, 030}^{3}
\end{array}\right) .
$$

The coefficient $b_{z, i j k}^{n}$ in the matrix $\Gamma$ of Eq. (9) is the magnetic $i j k$-inhomogeneity [as defined in Eq. (7)] created by the $n$th pair of rectangular loops of Fig. 1 when the applied set of currents is $\left\{I_{n}=1 \wedge I_{m}=0, \forall m \neq n\right\}$. Thus, Eq. (8) merely represents the superposition principle for the magnetic field generated by the source of Fig. 1 at the position of interest. The dependence of $\Gamma$ on $y_{0}$ has been made explicit in Eq. (9), emphasizing the variation of that matrix when choosing a different position.

\section{Measurement of $\Gamma$ and magnetic field compensation}

According to its definition, the $n$th column of $\Gamma\left(y_{0}\right)$ of Eq. (9) can be obtained experimentally by first applying some current to the $n$th pair of loops, while keeping the others with no current. The resulting magnetic field distribution along $\hat{u}_{y}$ is then measured in the vicinity of $\left(0, y_{0}, 0\right)$. Finally, with the measured distribution, the corresponding $B_{z, 0 j 0}$-inhomogeneities are evaluated. These must be normalized with respect to the current employed in the experiment. Repeating this procedure for all four independent currents of Fig. $1, \Gamma\left(y_{0}\right)$ is fully measured. With this, Eq. (8) can be inverted, and the appropriate set of currents, $\tilde{I}=\left(I_{0}, I_{1}, I_{2}, I_{3}\right)$, for a target field, $\tilde{B}=\left(B_{z, 0}, B_{z, 010}, B_{z, 020}, B_{z, 030}\right)$, can be determined as follows:

$$
\tilde{I}=\Gamma^{-1}\left(y_{0}\right) \cdot \tilde{B}
$$

For the geonium chip-as for any Penning trap-the most common configuration is the homogeneous field, defined by the target set $\tilde{B}=\left(B_{z, 0} \neq 0,0,0,0\right)$. Another very important configuration is the magnetic bottle, defined as $\tilde{B}=\left(B_{z, 0} \neq 0,0, B_{z, 020} \neq 0,0\right)$. The magnetic bottle is essential for determining the quantum state of the spin of a trapped electron/ion ${ }^{24}$ and is also the basis for developing a trapped electron as a quantum transducer of microwave photons.

\section{2. $\Gamma$ beyond the "long $L_{x}$ " approximation}

The vector potential terms $\vec{A}_{0}$ and $\vec{A}_{1}$ given in Eqs. (3) and (4) are always valid for the source of Fig. 1. However, when the "long $L_{x}$ " approximation does not hold, the higher order expansion terms deviate from the expressions given in Eqs. (5) and (6). In that case, it can be shown ${ }^{22,23}$ that, in particular, $\vec{A}_{2}$ $=\left(-\frac{1}{2 !} B_{z, 002} z^{2}\left(y-y_{0}\right)-\frac{1}{3 !} B_{z, 020}\left(y-y_{0}\right)^{3}\right) \hat{u}_{x}$. Hence, while within the mentioned approximation, the two second-order inhomogeneities are linked through ${ }^{22,23} B_{z, 020}=-B_{z, 002}$, beyond that approximation, these two quantities are usually still similar, $B_{z, 020} \simeq-B_{z, 002}$, but, in principle, independent of each other. Therefore, for a homogeneous field, they must be canceled separately. In that case, the target set becomes $\tilde{B}=\left(B_{z, 0}, B_{z, 010}, B_{z, 020}, B_{z, 002}, B_{z, 030}\right)$. In order to address this new set, containing now five quantities, we would need to expand the source of Fig. 1 with one extra pair of current loops. This would provide an additional independent current, which could be tuned to eliminate the extra inhomogeneity $B_{z, 002}$. Alternatively, one of the previous inhomogeneities can be abandoned, for instance, $B_{z, 030}$, and the target kept to only four quantities $\rightarrow \tilde{B}=\left(B_{z, 0}, B_{z, 010}, B_{z, 020}, B_{z, 002}\right)$. The set of currents $\tilde{I}$ and $\tilde{B}$ are still 
linked through Eq. (10); however, the matrix $\Gamma$ must be redefined to

$$
\Gamma^{\prime}\left(y_{0}\right)=\left(\begin{array}{cccc}
b_{z, 0}^{0} & b_{z, 0}^{1} & b_{z, 0}^{2} & b_{z, 0}^{3} \\
b_{z, 010}^{0} & b_{z, 010}^{1} & b_{z, 010}^{2} & b_{z, 010}^{3} \\
b_{z, 020}^{0} & b_{z, 020}^{1} & b_{z, 020}^{2} & b_{z, 020}^{3} \\
b_{z, 002}^{0} & b_{z, 002}^{1} & b_{z, 002}^{2} & b_{z, 002}^{3}
\end{array}\right) .
$$

The procedure for the measurement of $\Gamma^{\prime}$ in Eq. (11) is identical to the one discussed in Sec. II B 1 for $\Gamma$. The fourth row would seemingly require measuring the magnetic field distribution along the axial direction $\hat{u}_{z}$. However, $\Gamma^{\prime}$ can be determined by measuring the field only along $\hat{u}_{y}$, as described in detail in Sec. IV B.

\section{Expansion of the magnetic field source with further currents}

With the source introduced in Fig. 1 consisting of only four independent pairs of current loops, two options have been introduced: either using $\Gamma$, as defined in Eq. (9), or $\Gamma^{\prime}$ of Eq. (11). Depending on the choice, the set of tunable inhomogeneities will be different: either $\left(B_{z, 0}, B_{z, 010}, B_{z, 020}, B_{z, 030}\right)$ or $\left(B_{z, 0}, B_{z, 010}, B_{z, 020}, B_{z, 002}\right)$, respectively. If the first choice is made, then the currents can be tuned to deliver $B_{z, 020}=0$, which will automatically make $B_{z, 002}$ very small but not exactly equal to zero. Nevertheless, if the experiment requires $B_{z, 020}=B_{z, 002}=0$, then the matrix $\Gamma^{\prime}$ should be chosen. The condition $B_{z, 020}=B_{z, 002}=0$ can be then reached exactlywithin experimental boundaries-but at the expense of having $B_{z, 003}$ $\neq 0$. In our case, motivated by the use of the magnetic field source for ion trapping, since the effect of $B_{z, 003}$ upon the motion of the trapped particle is much weaker ${ }^{16}$ than the influence of $B_{z, 002}, \Gamma^{\prime}\left(y_{0}\right)$ of Eq. (11) is generally preferred.

The source of Fig. 1 can be expanded with further current loops, providing the possibility of eliminating increasing numbers of magnetic inhomogeneities. In general, a set of $n$ independent currents will define a $\Gamma$ matrix of rank $n \times n$, and this will provide the possibility of tuning or canceling any $n$ independent magnetic terms $B_{z, i j k}$, as defined in Eq. (7). Atomic physics measurements with trapped ions at the ppb level of precision show that magnetic inhomogeneities up to $B_{z, 004}$ might play a significant role at that level of accuracy. ${ }^{2}$ Within the "long $L_{x}$ " approximation, eliminating $B_{z, 004}$ would just require an extra fifth current loop for the source of Fig. 1. Beyond that approximation, compensating all inhomogeneities up to (and including) $\vec{A}_{4}$ in the series expansion of the vector potential will require the use of ten independent current loops. ${ }^{22,23}$ Since the compensation currents $\tilde{I}$ obey a linear system of equations, as shown in Eq. (10), they can be easily computed for increasing $n$. Within this article though, we concentrate on the basic case $n=4$.

\section{EXPERIMENTAL REALIZATION OF THE SOURCE}

We have fabricated a magnetic field source, as introduced in Fig. 1, with a set of four pairs of concentric current loops implemented with $\mathrm{NbTi}$ superconducting wires. These are enclosed within an aluminum frame, consisting of four symmetric pairs of rectangular-shaped casings. This metallic structure is shown in Fig. 3. Each casing serves as a spool around which the superconducting wire is wound, forming a rectangular loop. As shown in Fig. 3, the different spools are extractable such that winding the $\mathrm{NbTi}$ wire is done in each of them independently of the others. Each
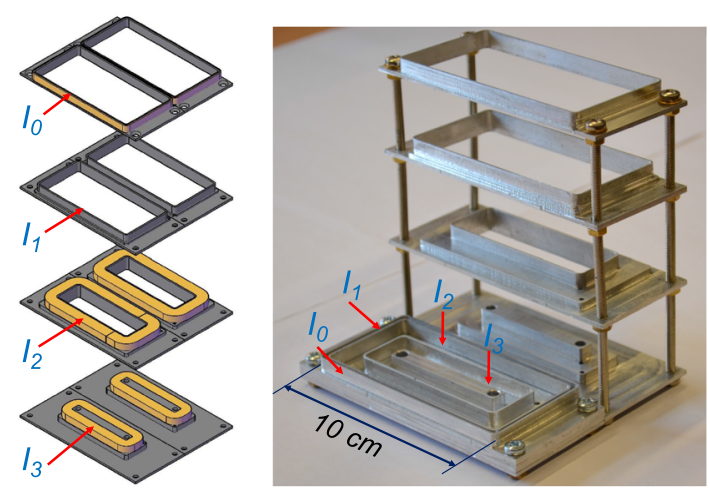

FIG. 3. The superconducting wires are confined inside an aluminum frame, comprising four pairs of symmetric rectangular containers.

casing in one loop-pair shares the same single piece of superconducting wire with its mirror-symmetric casing, that is, with no soldered joints between them. ${ }^{17}$ Once the wire winding has been completed, the containers are assembled on a plane, where all the different loops rest at the same height. Figure 4(a) shows the coplanar set of concentric, rectangular metallic casings before winding the wires. Figure 4(b) presents the complete source with the NbTi wires in place. The eight outer screws hold the whole structure together, while the four inner ones enable fixing it to the geonium chip trap and also to the calibration board discussed in Sec. IV A.

\section{A. Dimensions and materials}

The dimensions of the source are given in Fig. 5. The total length in the $\hat{u}_{x}$ direction is $L_{x}=98.6 \mathrm{~mm}$, while in $\hat{u}_{z}$, it is $2 \times L_{z}$ $=99.2 \mathrm{~mm}$. The gap between the two symmetric sections is negligible, $\Delta z \simeq 0$. The respective widths of the rectangular current loops are $a_{0}=0.8 \mathrm{~mm}, a_{1}=1.2 \mathrm{~mm}, a_{2}=8.8 \mathrm{~mm}$, and $a_{3}=7.5 \mathrm{~mm}$. The gap between any two adjacent concentric loops is $g_{0}=1.0 \mathrm{~mm}$. The thickness of the source is $6 \mathrm{~mm}$ (wires only).

The values of $a_{0}, a_{1}, a_{2}$, and $a_{3}$ have been optimized (within the constraint of given fixed $L_{x}$ and $L_{z}$ ) for minimizing the amplitude of the currents required for an homogeneous field at $y_{0}=1.6 \mathrm{~mm}$ (measured from the magnetic field source's surface). This $y_{0}$ corresponds to the optimal trapping position ${ }^{16}$ of the geonium chip ${ }^{17}$ of $0.8 \mathrm{~mm}+0.7 \mathrm{~mm}$ chip's thickness $+0.1 \mathrm{~mm}$ buffer layer between

a)

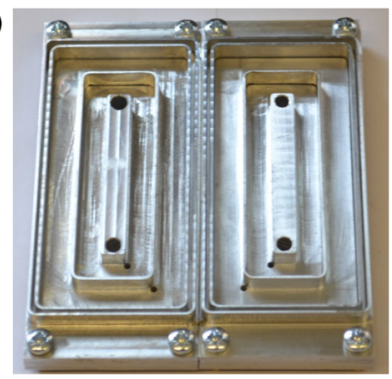

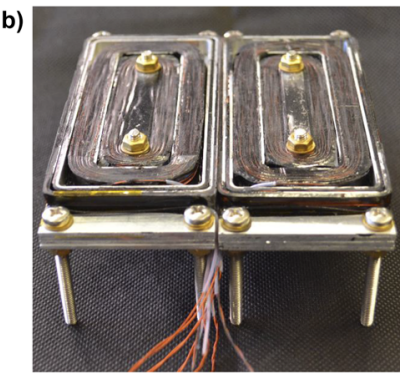

FIG. 4. (a) Aluminum structure with rectangular casings. (b) Complete magnetic field source. 


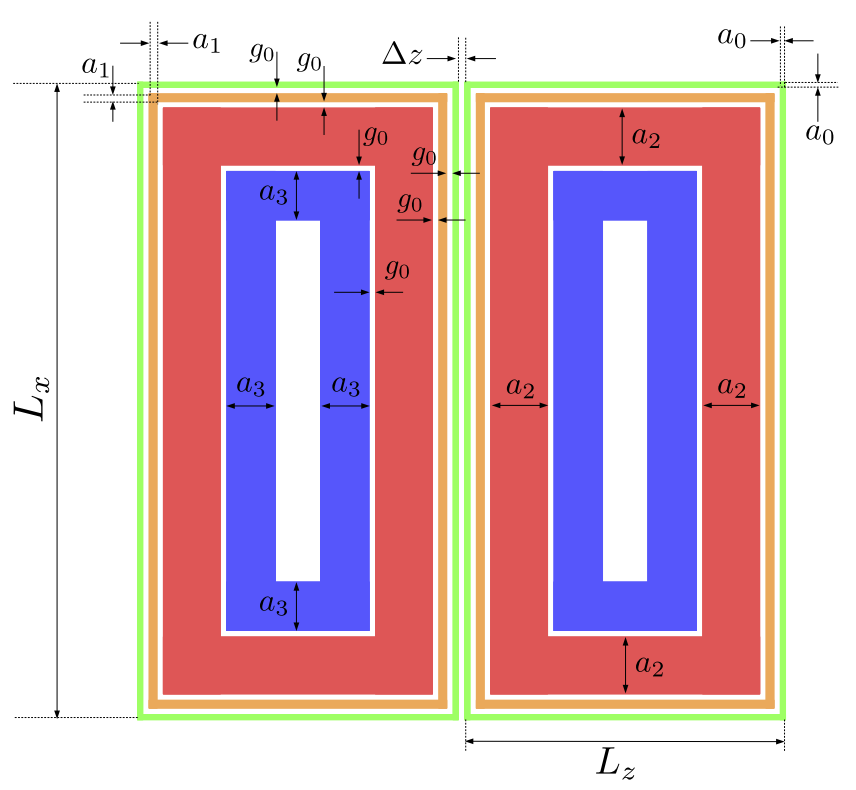

FIG. 5. Dimensions of the source.

the chip and the magnetic field source. Furthermore, we have used a total length of about $4 \mathrm{~km}$ of insulated, copper-stabilized, monofilament NbTi wire (Supercon T48B-M). ${ }^{26}$ This wire has a diameter of $0.127 \mathrm{~mm}$-including the outer insulation-with a superconducting core of $0.062 \mathrm{~mm}$ diameter. It is rated to sustain a critical current of up to $11 \mathrm{~A}$ in an ambient field of $3 \mathrm{~T}^{26}$ The wires have been wound with a standard winding machine. The approximate number of turns is $120,225,1995$, and 1982 for each section of the currents $I_{0}, I_{1}, I_{2}$, and $I_{3}$, respectively. The wires are fixed and mechanically stabilized to the aluminum containers with cryogenic epoxy. All metallic sharp corners in Figs. 3 and 4 have been smoothly rounded for avoiding cutting and damaging the NbTi superconductors. Small holes in the bottom surface of the aluminum casings [see Fig. 4(a)] allow for threading the single piece of wire used in each loop through to its mirror-symmetric partner in the other section. These holes also align the input and output sides of the wires. Outside the rectangular casings, any incoming and outgoing wires are bundled in twisted pairs of counter-propagating currents (input-output) such that the undesired magnetic field they create is canceled or strongly reduced. This is verified experimentally in the measurements presented in Sec. IV.

\section{B. Geonium chip cryostat}

In order to operate the magnetic field source below the $T_{c} \sim 9 \mathrm{~K}$ of $\mathrm{NbTi}$, we use a two-stage pulse-tube cryocooler. This is shown in Fig. 6. The cold head is a Sumitomo RP062 model, providing $30 \mathrm{~W}$ of cooling power at the first $(60 \mathrm{~K})$ stage and $0.5 \mathrm{~W}$ cooling power at the second $(4 \mathrm{~K})$ stage. The currents for the magnetic field source are delivered from outside the cryostat by bench-top supplies. The source is designed for a Penning trap experiment, which is very sensitive to radio frequency noise. ${ }^{27,28}$ It is therefore necessary to filter the currents to prevent the noise from coupling into the trapped particles' detection circuit. The noise filters consist of a low-pass RC filter and a common-mode inductive filter, for each current, and they

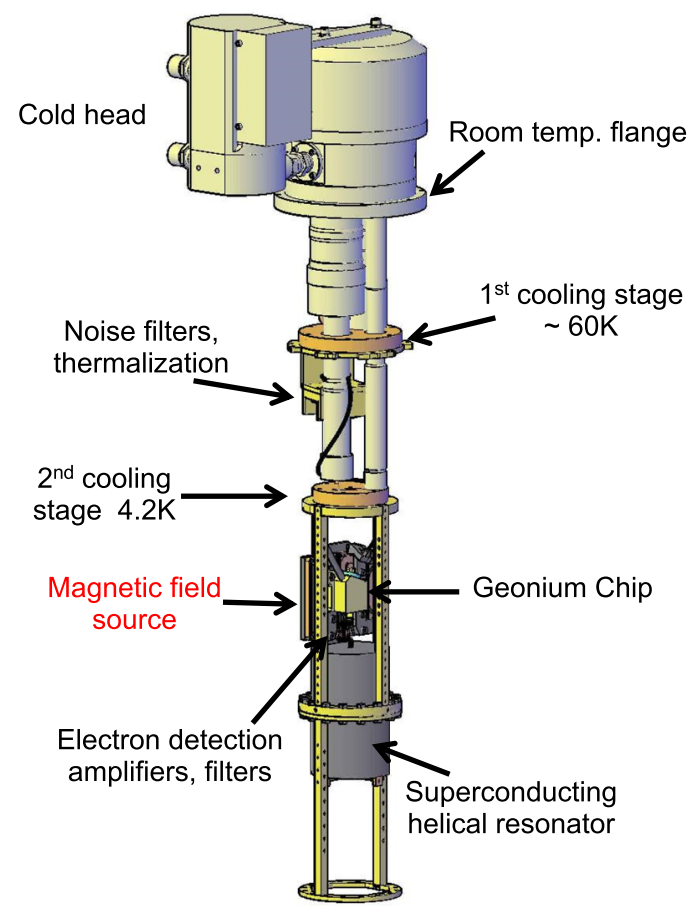

FIG. 6. Sketch of the geonium chip cryostat. The volume within the cryostat vessel is magnetically shielded with a $\mu$-metal foil (not shown).

are housed outside the cryostat. At values greater than $10 \mathrm{~A}$, significant heat is generated in the filters. Hence, these are water-cooled to remove the heat and prevent it from flowing into the cryostat. Without water-cooling, the heat flows down the wires into the $4 \mathrm{~K}$ region, raising the base temperature of the system and leading to an increased probability of "quenching" the magnetic field source.

\section{Wiring and thermalization}

The $0.5 \mathrm{~W}$ cooling power of the second stage can be easily overwhelmed by the sum of the thermal conductivity down the wires plus the resistive heating caused by the electric currents. To avoid these, it is critical to implement proper thermal anchoring of the wires to the cold-head and to minimize the resistive ohmic dissipation. The magnetic field currents are first fed into the cryostat's vacuum chamber using conventional copper wires. These wires connect the room-temperature flange (see Fig. 6) to the first $60 \mathrm{~K}$ stage of the pulse-tube. The length and diameter of those wires is optimized for $10 \mathrm{~A}$, according to well-known methods. ${ }^{29,30}$ At the $60 \mathrm{~K}$ stage, the wires are then attached by solder tags to thermally anchored busbars. ${ }^{17}$ These are shown in Fig. 7(a). This allows for each wire to thermalize with the $60 \mathrm{~K}$ stage, while also providing a break in the wire. In general, it is best practice to maximize the footprint of the solder tags and bus-bar (space allowing) to minimize contact resistance and maximize heat transfer to the cooling stage. The solder tag has a footprint of $1 \mathrm{~cm}^{2}$ and the bus-bar $4.25 \mathrm{~cm}^{2}$ with a thickness of $5 \mathrm{~mm}$. They are attached together with screws. Both the tags and the bus-bars have been gold plated to inhibit corrosion, which otherwise would decrease thermal transfer between the tag and the bar. 

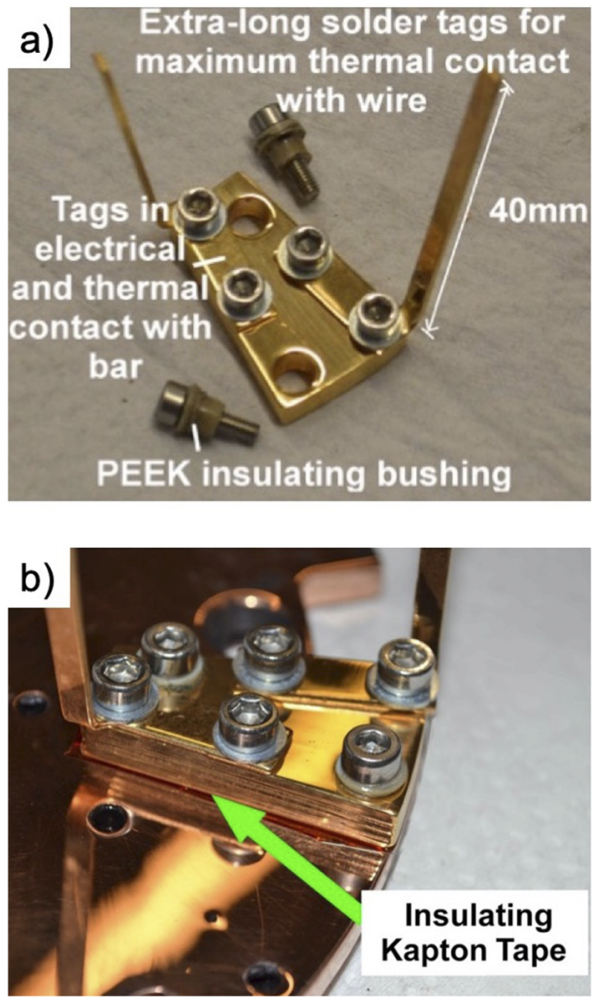

FIG. 7. (a) Gold-plated thermalization bus-bar with solder-tag. The mounting screws are provided with PEEK plastic bushings to prevent electrical contact with the bar. (b) Thermalization block attached to the pulse-tube first stage faceplate. The block is electrically isolated from the bulk with Kapton tape.

As shown in Fig. 7(b), the bars are electrically insulated from ground (the $60 \mathrm{~K}$ cold plate) by Kapton tape.

\section{Geonium chip cryostat}

The first stage temperature of $60 \mathrm{~K}$ enables the use of high temperature superconductors to further transport the currents from there to the second cooling stage at $4 \mathrm{~K}$. For this purpose, we use HTS tape ${ }^{31}$ made of ReBCO with a critical temperature of $\mathrm{T}_{c} \sim 90 \mathrm{~K}$. The tape has a width of $2 \mathrm{~mm}$ and sustains a critical current of $40 \mathrm{~A}$. Using HTS tape offers a number of advantages over conventional copper wiring. For a given current, copper wire must be chosen with an optimal length and diameter, which minimizes the sum of ohmic and thermal conductive heating. Since the ohmic heating is eliminated when using HTS tape, there are no constraints as to any required optimal cross section. This allows for more flexibility in the system design, and HTS tape can be generally used with a much smaller cross section than copper wiring with an equivalent current rating. In the case of our system, designed for $10 \mathrm{~A}$ current operation, an optimal cross section of $0.5 \mathrm{~mm}^{2}$ would be required for copper. $^{29}$ This must be compared to the bare $0.08 \mathrm{~mm}^{2}$ for $2 \mathrm{~mm}$ ReBCO tape we use. ${ }^{31}$ Furthermore, since the HTS tape is flat, the surface area per unit length is also greater, thus allowing better heat sinking along its length. The thermalization measures are repeated at the $4 \mathrm{~K}$ plate to join the HTS tape and NbTi wire; however, due to space constraints, the tags in this region have a reduced footprint of $0.6 \mathrm{~cm}^{2}$, and the $4 \mathrm{~K}$ bus-bar has a reduced footprint of $3 \mathrm{~cm}^{2}$, still with a $5 \mathrm{~mm}$ thickness. The solder tags also retain the $40 \mathrm{~mm}$ joint length. The tape is not without its downsides; it is much more expensive than copper wire, it is also easily damaged, and care must be taken while soldering to avoid destroying the superconductive properties. The flat form and minimum bend radius can make wiring difficult in confined spaces, such as our cryostat (Fig. 6).

\section{Quench protection}

For the case of a quench, the magnetic field source is first provided with passive thermal protection in the form of heat sink "thick wires" and bypass diodes. While the source topology is conceived for future persistent current operation, presently it is driven with external current supplies. The mentioned passive protection would be quickly overwhelmed if the currents continued to run while quenching. Therefore, an automated protection procedure has been implemented to protect the magnetic field source in the event of a suspected quench. This uses a LabVIEW program, which identifies quenches by measuring rapid changes in the temperature of the source with time. The temperature in the cryostat is measured every $200 \mathrm{~ms}$, at three different spots, with a Lakeshore 218 temperature controller. If the maximum permitted value for the temperature or the rate of temperature increase is exceeded, the control program identifies that a quench has begun. In that case, all four outputs of the Rohde and Schwarz HMP4040 current supplies are turned off using a single Standard Commends for Programmable Instruments command, with an estimated time delay of less than $200 \mathrm{~ms}$. The inductance of the largest coils is only a few $\mathrm{mH}$, and the magnetic energy is dumped through the bypass diodes, making it safe to switch the current off very rapidly, without the need of ramping it slowly down.

This simple procedure has some limitations, such as the reaction speed of the LabVIEW program and devices, and the possibility of wrongly identifying as a quench a simple transit noise spike in the cryogenic temperature sensors. We have experienced multiple quenches, but despite the aforementioned limitations, the magnetic field source has remained undamaged as a result of the active and passive quench protection measures.

\section{CALIBRATION AND TEST OF THE SOURCE}

In order to characterize the magnetic field source presented in Fig. 4 and test its performance, we first need to measure the matrix $\Gamma\left(y_{0}\right)$ of Eq. (9). We refer to the process of measuring that matrix as "calibration" of the source. The calibration is performed with the source in the superconducting state at $4 \mathrm{~K}$. While the source can also be calibrated at room temperature, the currents distribute differently within the $\mathrm{NbTi}$ wires at $4 \mathrm{~K}$ than at $300 \mathrm{~K}$. In the latter case, most of the current runs along the copper cladding, which holds the inner NbTi filament. ${ }^{26}$ This is due to the lower resistivity of copper than $\mathrm{NbTi}^{29}$ at room temperature. Thus, the effective cross section of the wires varies from room temperature to $4 \mathrm{~K},{ }^{30}$ and so does the created magnetic field for a given set of currents. This together with the Meissner-effect result in the calibration matrix, $\Gamma\left(y_{0}\right)$, being different when measured at $4 \mathrm{~K}$ or at $300 \mathrm{~K}$. While calibrating at room temperature is technically easier, we have performed the calibration at $4 \mathrm{~K}$, and this is reported in Secs. IV A-IV C. 


\section{A. Array of Hall-effect magnetic field sensors}

The measurement of the magnetic field along the vertical axis $\hat{u}_{y}$ is done by means of an array of Hall-effect sensors ${ }^{32}$ provided by the private company Arepoc. It is shown in Fig. 8(a). The array consists of seven sensors, all fabricated on a single chip and sharing the same bias current, as sketched in Fig. 8(b). The sensors are uniaxial, and they measure the magnetic field component normal to the surface of the chip, which corresponds to the $\hat{u}_{z}$ axis of Fig. 1. The sensor-sensor spacing amounts to $0.5 \mathrm{~mm}$ between the center of each active region of each sensor. They have been individually calibrated for cryogenic use at $4 \mathrm{~K}^{32}$ In the presence of a magnetic field, the resulting Hall voltage of each sensor is measured by a precision voltmeter. Each magnetic field measurement results from the average of several hundred Hall voltage measurements during a few seconds. Moreover, the sense of the bias current ("upward" or "downward," see Fig. 8) is reversed, and the final magnetic field reading is obtained from the average of the measurements with both current senses. ${ }^{33}$ This eliminates the ohmic voltage drop across the sensors and provides a "clean" Hall voltage measurement.

As illustrated in Fig. 8(c), the chip with the Hall sensors is installed on a printed circuit board (PCB) calibration board. This is specifically designed to align the magnetic sensors along the vertical $\hat{u}_{y}$ axis. ${ }^{33}$ The board has a thickness of $0.5 \mathrm{~mm}$ and is fixed directly on top of the magnetic field source with the screws shown in Fig. 9. The geonium chip has a thickness of $0.8 \mathrm{~mm}$, and this difference must be taken into account when specifying the calibration position $y_{0}$. Furthermore, a fiducial cross cut has been patterned on the PCB with a computer numerical control (CNC) machine, ${ }^{34}$ allowing for a positioning accuracy of $\pm 100 \mu \mathrm{m}$. The cross cut indicates the center of the magnetic field source $x=0, z=0$ (see Fig. 1), which also coincides with the center of the geonium chip. A macor block is used to stabilize the array of Hall sensors and fix it at the precise location marked by the cross cut.
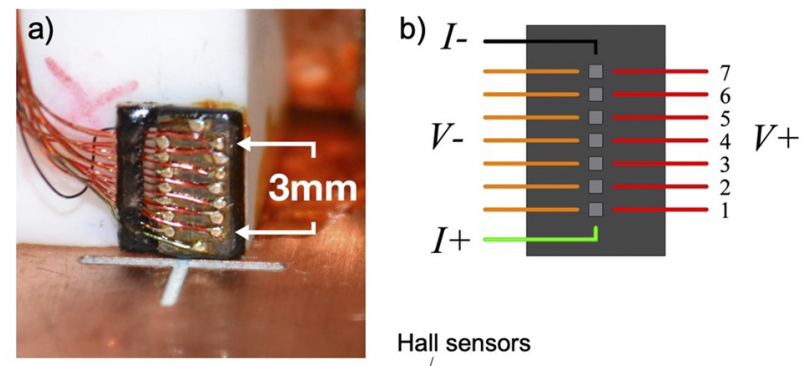

c)

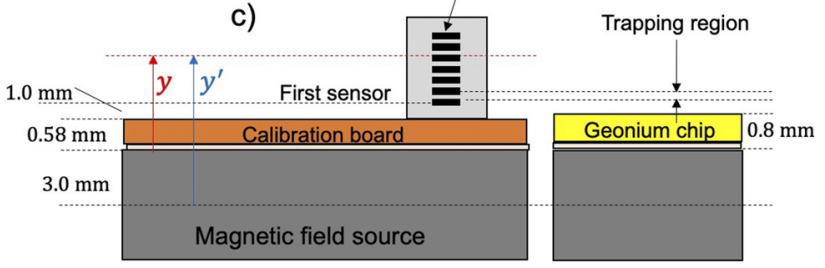

FIG. 8. (a) Array of Hall-effect sensors in a chip. This allows for measuring $B_{z}$ at seven fixed values of the height $y$. (b) Sketch of the relative positions and wiring configuration of the seven Hall sensors. (c) Comparison of the sensors' positions with the range of $y$ values of interest for the geonium chip.

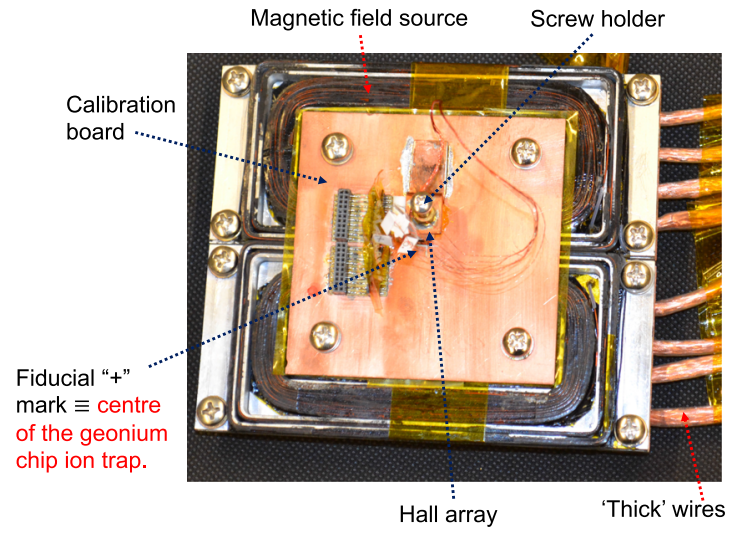

FIG. 9. Photo of the magnetic field source and calibration board.

Further features about the design, fabrication, and alignment of the calibration board have been described in detail elsewhere.

\section{B. Measurement of the calibration matrix $\Gamma\left(y_{0}\right)$}

As explained in Sec. II B $1, \Gamma\left(y_{0}\right)$ is obtained by measuring the magnetic field distribution around the position $\left(0, y_{0}, 0\right)$ created individually by each current $I_{n}$ of the source, with all other currents off. The current is delivered by a Rohde and Schwarz HMP4040 precision current supply, where each $I_{n}$ has its own specific source. The calibration is done with each current set at $1 \mathrm{~A}$. Higher currents could be used, which might then include possible non-linear effects in the calibration, for instance, due to magnetic saturation of materials within the cryostat. However, no such non-linear effects have been observed around the maximum operation currents $10 \mathrm{~A}-12 \mathrm{~A}$. With $I_{n}$ on, the array of sensors described in Sec. IV A measures seven magnetic field values $B_{z}(y)$, one for every single height $y$ with one Hall sensor (Fig. 8). Figure 10 shows the results for each single current measurement.

In Fig. 10, each set of measured magnetic fields for one current $I_{n}$ has been fitted to a theoretical curve $B_{z}^{n}(0, y, 0)$. The latter is obtained from Biot-Savart's law, as given in the following equation:

$$
\vec{B}^{n}(\vec{r})=\frac{\mu_{0}}{4 \pi} \oint d V^{\prime} \frac{\vec{J}_{n} \times\left(\vec{r}-\vec{r}^{\prime}\right)}{\left|\vec{r}-\vec{r}^{\prime}\right|^{3}} .
$$

In Eq. (12), the region of integration for each current density $J_{n}$, corresponding to the current $I_{n}$, is modeled, as shown in Fig. 11(a). Thus, the theoretical formulas of Eq. (12) assume a homogeneous current density distribution across the normal section of the source, $J=I / A$. This is illustrated in Fig. 11(b). Moreover, the Biot-Savart functions do not take into account the Meissner effect. However, they are evaluated/fitted at distances $\geq 1.5 \mathrm{~mm}$ above the $\sim 100 \mu \mathrm{m}$ thin $^{26}$ superconducting wires, where the Meissner effect can be neglected in the first instance.

As illustrated in Fig. 11(c), the current $I_{n}$ is not homogeneously distributed across the normal area of the source. In reality, that area is crossed by the thin NbTi wires, all of which carry the same current $I_{n}$. Hence, as the main free parameter for the fits of Fig. 10, we have used the effective current density $J_{n}$. The results are $J_{0}=25.54$ $\pm 0.05, J_{1}=31.37 \pm 0.06, J_{2}=37.73 \pm 0.07$, and $J_{3}=43.93 \pm 0.10$, all in 


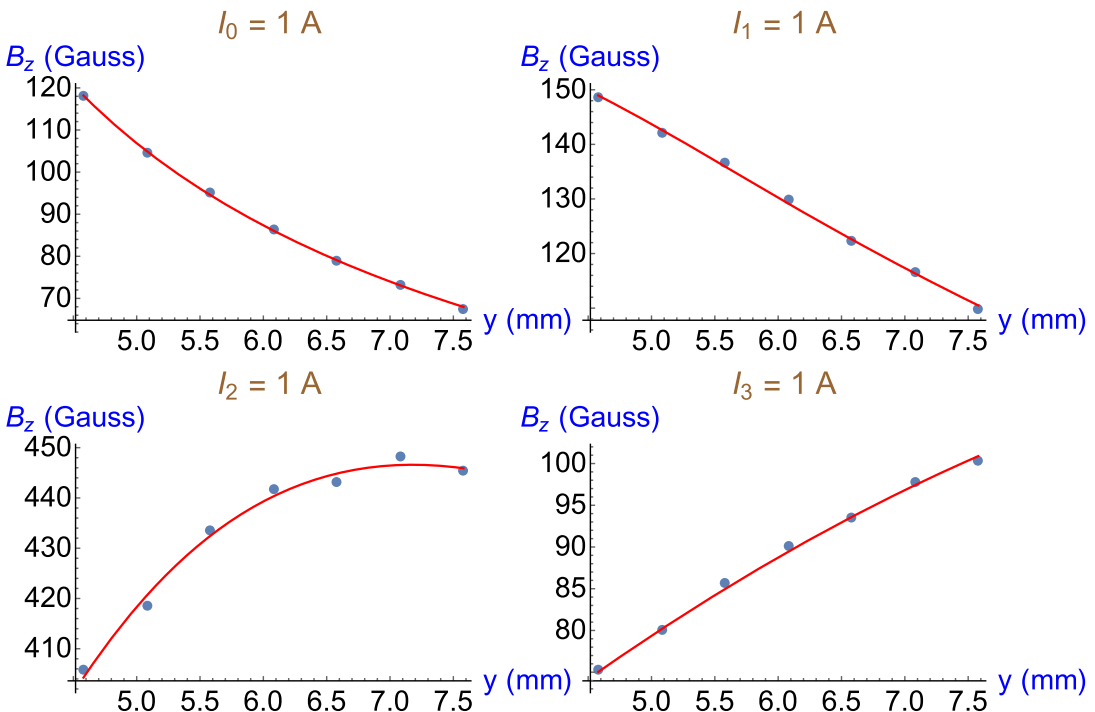

FIG. 10. Magnetic field created by each current $I_{n}$ when $1 \mathrm{~A}$ is applied, with $I_{m}$ $=0, \forall m \neq n$. The coordinate $y$ is the vertical distance with respect to the center of the magnetic field source $\left[\equiv y^{\prime}\right.$ in Fig. 8(c)]. Each continuous curve is the fit of the experimental data (dots) to their theoretical $B_{z}^{n}(0, y, 0)$ function. The statistical error bars are much smaller than the scale of the graphs.

$\mathrm{A} / \mathrm{mm}^{2}$. Since the applied current $I_{n}=1 \mathrm{~A}$ is known, the fits actually deliver the value of the effective cross sectional area, $A_{\text {effective }}^{n}$ (with $J_{n}=I_{n} / A_{\text {effective }}^{n}$ ), for each current $n \in[0,3]$. The values obtained for $A_{\text {effective }}^{n}$ are in very good agreement with the ones expected from the dimensions of the source and the number of turns of NbTi wire used. $^{33}$

As the free fitting parameter, we have also used the relative orientation of the magnetic field source and the array of Hall sensors. The fits indicated an average tilt angle between both vertical axes of the source and calibration board of $(6.6 \pm 1.8)^{\circ}$. Direct inspection is not practicable at $4 \mathrm{~K}$, with the system inside the cryostat vessel. It was therefore not possible to mechanically confirm such a tilt angle and correct for it. Repeating the measurement of Fig. 10 at $300 \mathrm{~K}$ delivered also the same "tilt angle." However, rather than a tilt angle between the source and the calibration board, it is more

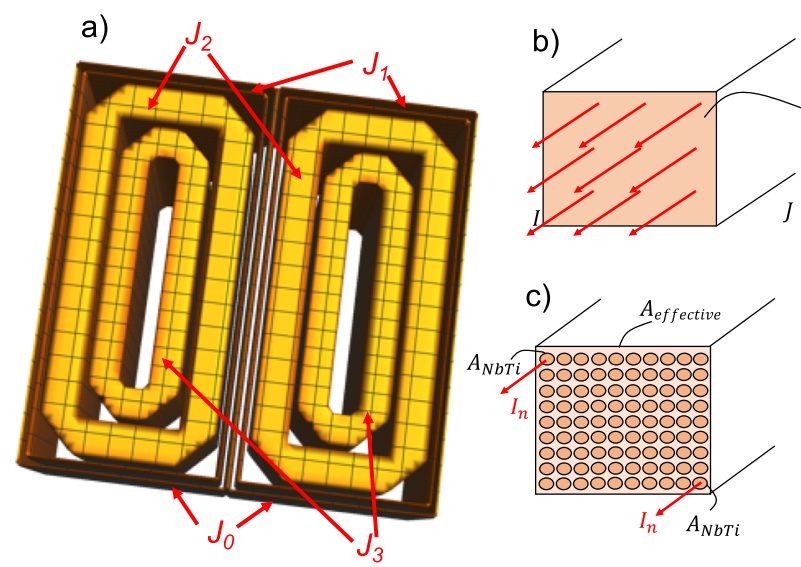

FIG. 11. (a) Integration regions for computing each theoretical $B_{z}^{n}$ function, $n \in[0,3]$. (b) Homogeneous current distribution. (c) NbTi thin wires passing through the normal area. The effective cross section $A_{\text {effective }}$ for each current $I_{n}$ is obtained from the fits of Fig. 10. probable that the NbTi wire wrapped around the rectangular spools of Fig. 3 suffered a slight inclination with respect to the spools during the winding procedure. ${ }^{17}$ Rewinding the wire for $I_{2}$ eliminated the "tilt angle" for that current, while it was still visible in the others $I_{0}, I_{1}$, and $I_{3}$. Hence, in the rest of this article, we assume a fixed, effective "tilt angle" for those currents and zero for $I_{2}$.

\section{Results for $\Gamma\left(y_{0}\right), \Gamma^{\prime}\left(y_{0}\right)$}

With the experimentally determined $B_{z}^{n}(\vec{r})$ functions of Fig. 10, we can obtain the calibration matrix $\Gamma\left(y_{0}\right)$. We only need to compute the Taylor series expansion of each fitted function around the point of interest, $\left(0, y_{0}, 0\right) \rightarrow B_{z}^{n}(0, y, 0)=B_{z}^{n}\left(y_{0}\right)+\left.\frac{\partial B_{z}^{n}}{\partial y}\right|_{y_{0}}\left(y-y_{0}\right)+$ $\left.\frac{1}{2} \frac{\partial^{2} B_{z}^{n}}{\partial y^{2}}\right|_{y_{0}}\left(y-y_{0}\right)^{2}+\cdots$. Since $B_{z}^{n}(0, y, 0)$ has been measured with $I_{n}=1 \mathrm{~A}$, its series expansion coefficients directly deliver the elements of the $n$th column of $\Gamma\left(y_{0}\right)$, as defined in Eq. (9).

As a concrete example, we have obtained $\Gamma$ at $y_{0}=1.45 \mathrm{~mm}$ above the geonium chip surface [see Fig. 8(c)]. The result is given in the following equation:

$$
\begin{aligned}
\Gamma & \left(y_{0}=1.45 \mathrm{~mm}\right) \\
& =\left(\begin{array}{cccc}
101.17 \pm 0.14 & 140.41 \pm 0.23 & 424.6 \pm 0.5 & 81.83 \pm 0.19 \\
-21.52 \pm 0.24 & -13.43 \pm 0.26 & 26.0 \pm 1.0 & 9.71 \pm 0.25 \\
4.86 \pm 0.10 & -0.54 \pm 0.05 & -9.1 \pm 0.5 & -0.64 \pm 0.03 \\
-1.18 \pm 0.04 & 0.61 \pm 0.05 & 0.84 \pm 0.06 & -0.0115 \pm 0.0009
\end{array}\right) .
\end{aligned}
$$

The $\Gamma$ matrix of Eq. (13) is given in Gauss $/ \mathrm{mm}^{j}$, where $j=0$ for the first row, $j=1$ for the second, etc. The errors in the matrix elements result from the propagation of the errors of the fitting parameters of Fig. 10. In general, the relative error grows with the order of the derivative.

The determination of the $\Gamma^{\prime}$ matrix, as defined in Eq. (11), is straightforward: we simply need to compute the series expansion 
$B_{z}^{n}\left(0, y_{0}, z\right)=B_{z}^{n}\left(y_{0}\right)+\left.\frac{1}{2} \frac{\partial^{2} B_{z}^{n}}{\partial z^{2}}\right|_{y_{0}} z^{2}+\left.\frac{1}{4 !} \frac{\partial^{4} B_{z}^{n}}{\partial z^{4}}\right|_{y_{0}} z^{4}+\cdots$, where the odd derivatives have vanished due to the symmetry of the magnetic field source along the $z$ axis. This symmetry has been checked experimentally with the source at room temperature, ${ }^{33}$ where a movable Hall sensor can be placed with $100 \mu \mathrm{m}$ precision or better at several $\pm z$ positions around $\left(0, y_{0}, 0\right)$. The measurements confirm the vanishing values of $\left.\frac{\partial B_{z}^{n}}{\partial z}\right|_{y_{0}}$ and $\left.\frac{\partial^{3} B_{z}^{n}}{\partial z^{3}}\right|_{y_{0}}$ within error bars. Hence, at $y_{0}=$ $1.45 \mathrm{~mm}$, the result for $\Gamma^{\prime}$ is (in Gauss $/ \mathrm{mm}^{j}$ )

$$
\begin{aligned}
\Gamma^{\prime}\left(y_{0}\right. & =1.45 \mathrm{~mm}) \\
& =\left(\begin{array}{cccc}
101.17 \pm 0.14 & 140.41 \pm 0.23 & 424.6 \pm 0.5 & 81.83 \pm 0.19 \\
-21.52 \pm 0.24 & -13.43 \pm 0.26 & 26.0 \pm 1.0 & 9.71 \pm 0.25 \\
4.86 \pm 0.10 & -0.54 \pm 0.05 & -9.1 \pm 0.5 & -0.64 \pm 0.03 \\
-6.10 \pm 0.1 & 0.73 \pm 0.05 & 9.2 \pm 0.5 & 0.92 \pm 0.03
\end{array}\right) .
\end{aligned}
$$

As discussed in Sec. II B 2, for an infinitely long $L_{x}$ source, the third and fourth rows of $\Gamma^{\prime}$ should be identical, but with opposite signs. That approximation is now corrected for with the use of $\Gamma^{\prime}$ of Eq. (14). This shows that those rows are generally similar (with inverted signs) but not identical. The rank of $\Gamma^{\prime}$ is therefore four, and it can be inverted, thus delivering the required currents for a target magnetic field $\tilde{B}$. Using Eq. (10), we can now determine the set of currents for a homogeneous $0.5 \mathrm{~T}$ field at $y_{0}=1.45 \mathrm{~mm}$ as follows:

$$
\tilde{I}=\left(\begin{array}{c}
I_{0}=6.4 \pm 0.2 \\
I_{1}=12.1 \pm 0.6 \\
I_{2}=0.75 \pm 0.25 \\
I_{3}=29.2 \pm 0.50
\end{array}\right) \text { A, } \tilde{I}^{\prime}=\left(\begin{array}{c}
I_{0}=7.6 \pm 0.3 \\
I_{1}=10.1 \pm 0.5 \\
I_{2}=1.6 \pm 0.6 \\
I_{3}=26.5 \pm 0.5
\end{array}\right) \text { A. }
$$

\section{Optimization of the currents}

The currents obtained with matrices 13 and 14 can be optimized by leaving one of the inhomogeneities floating, for instance, $B_{z, 030}$ in the former case. The target $\tilde{B}$ is then decreased by one element, leaving only three quantities, $\tilde{B}_{\text {reduced }}=\left(B_{z, 0}, B_{z, 010}, B_{z, 020}\right)$, to be shaped with four independent currents. Hence, Eq. (10) becomes a linear system of only three equations with four independent variables. The solution is an infinite set of currents, which deliver the desired target $\tilde{B}_{\text {reduced }}$. This infinite set of currents can be investigated by plotting three of the currents as a function of the fourth one. "Optimizing" the currents means finding the set $\left(I_{0}, I_{1}, I_{2}, I_{3}\right)$ that makes the largest element of the set (in absolute values) minimal. An example of such "optimization" of the currents is shown in
Fig. 12. The target is a homogeneous $0.5 \mathrm{~T}$ field at $y_{0}=1.45 \mathrm{~mm}$. The optimized set of currents is

$$
\tilde{I}=\left(\begin{array}{c}
I_{0}=13.1 \pm 0.4 \\
I_{1}=0.165 \pm 0.008 \\
I_{2}=6.0 \pm 2.0 \\
I_{3}=13.1 \pm 0.4
\end{array}\right), \quad \tilde{I}^{\prime}=\left(\begin{array}{c}
I_{0}=11.3 \pm 0.4 \\
I_{1}=2.1 \pm 0.1 \\
I_{2}=6.2 \pm 2.0 \\
I_{3}=11.3 \pm 0.4
\end{array}\right) \text { A. }
$$

Comparing the currents in Eq. (16) to those in Eq. (15), it is clear that the largest current of the set has dropped by a factor of two or more. This dramatically decreases the thermal load upon the $4 \mathrm{~K}$ stage of the cryostat, allowing for homogeneous magnetic fields of $0.5 \mathrm{~T}$. The current optimization has the downside of leaving $B_{z, 030}$ (or $B_{z, 020}$ ) floating. However, as discussed in Sec. IV E, the measured residual value of $B_{z, 030}$ has a negligible effect on the trapped electron's motional eigenfrequencies.

\section{E. Test of the magnetic field source}

Figure 13 shows the result for a target magnetic field $\tilde{B}_{\text {reduced }}$ $=\left(B_{z, 0}=0.3 \mathrm{~T}, B_{z, 010}=0, B_{z, 020}=0\right)$. We have used the set of currents $\tilde{I}$ given in Eq. (16) rescaled for this target. The residual inhomogeneities are obtained with the same procedure of Sec. IV B. As fitting parameters, we now use only three current densities, $J_{0}, J_{2}, J_{3}$. The reason is to keep the amount of fitting parameters as small as possible, considering the limited number of data available. Moreover, taking into account the small value of $I_{1} \leq 0.1 \mathrm{~A}$ [see Eq. (16)], the corresponding density $J_{1}$ has been fixed (with the value of $A_{\text {effective }}^{1}$ found in Sec. IV C) and not fitted. As seen in Fig. 13(a), the analysis is first done with all seven sensors data. The fit is then repeated in Fig. 13(b) excluding sensors 2 and 5 [see Fig. 8(b)]. As will be explained in Sec. IV F, for magnetic fields as strong as in Fig. 13, those two sensors appear to suffer a small offset with respect to the others. However, it is not possible to distinguish which sensors are the "right" ones. Therefore, we average the results of both fits obtained in Figs. 13(a) and 13(b). The measured residual inhomogeneities are listed in the following equation:

$$
\left(\begin{array}{cc}
B_{z, 0}=3022.5 \pm 0.7 & \mathrm{G} \\
B_{z, 010}=-2.0 \pm 0.8 & \mathrm{G} / \mathrm{mm} \\
B_{z, 020}=0 \pm 2 & \mathrm{G} / \mathrm{mm}^{2} \\
B_{z, 030}=-8 \pm 2 & \mathrm{G} / \mathrm{mm}^{3}
\end{array}\right)
$$

Figure 14 shows the results for a target magnetic field $\tilde{B}_{\text {reduced }}$ $=\left(B_{z, 0}=0.3 \mathrm{~T}, B_{z, 010}=0, B_{z, 002}=0\right)$. In this case, we have used the currents $\tilde{I}^{\prime}$ of Eq. (16), rescaled for a $0.3 \mathrm{~T}$ target. The analysis is iden-
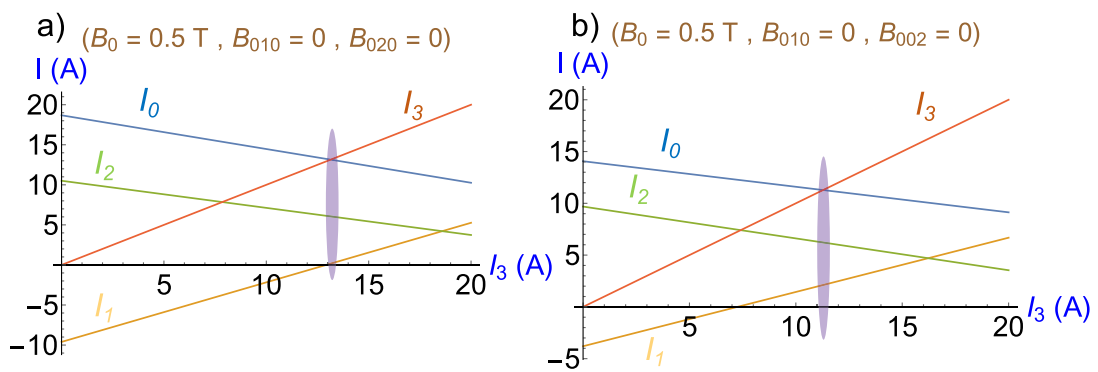

FIG. 12. (a) Optimization of the currents for $\left(B_{z, 0}, B_{z, 010}, B_{z, 020}\right)$. Around $I_{3} \sim$ $13 \mathrm{~A}$, the current set becomes optimal. (b) Optimization for $\left(B_{z, 0}, B_{z, 010}, B_{z, 002}\right)$ Optimization is achieved around $I_{3} \sim$ $11 \mathrm{~A}$. 
a) $B_{\mathrm{z}}(\mathrm{G}) \quad B_{0}=0.3 \mathrm{~T}, B_{010}=0, B_{020}=0$

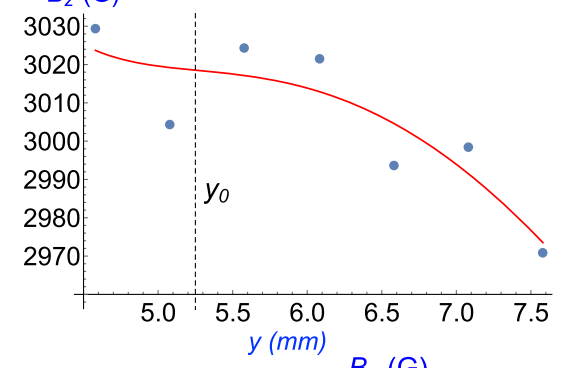
$B_{z}(\mathrm{G})$ 3500
3000
2500
2000
1500
1000
500 b) $B_{0}=0.3 T, B_{010}=0, B_{020}=0$

$B_{z}(\mathrm{G})$

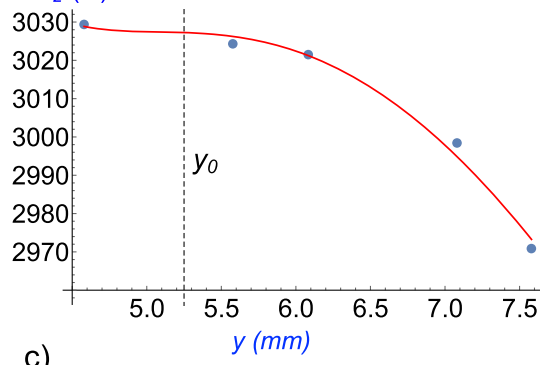

FIG. 13. (a) Result magnetic field at $y_{0}=$ $1.45 \mathrm{~mm}$ (above geonium chip's surface) calibrated with $\Gamma$ of Eq. (13). The target $\tilde{B}_{\text {reduced }}$ is shown on top of the graph. Function fitted with all seven sensors. (b) Result when the Hall-sensors 2 and 5 are removed from the fit. (c) Plot of the fitted function in a bigger range of heights. In all graphs, the variable $y$ is given with the origin in the center of the magnetic field source $\left[\equiv y^{\prime}\right.$ in Fig. 8(c)]. tical as in the previous case. The measured residual inhomogeneities are given in the following equation:

$$
\left(\begin{array}{cc}
B_{z, 0}=3009.5 \pm 0.7 & \mathrm{G} \\
B_{z, 010}=-0.4 \pm 0.6 & \mathrm{G} / \mathrm{mm}^{2} \\
B_{z, 020}=-7 \pm 2 & \mathrm{G} / \mathrm{mm}^{2} \\
B_{z, 030}=-5 \pm 2 & \mathrm{G} / \mathrm{mm}^{3}
\end{array}\right)
$$

\section{F. Comparison of the two configurations}

In order to check the results obtained in Eqs. (17) and (18), we subtract the experimental dataset of Fig. 13(a) minus the set of Fig. 14(a). The outcome of this subtraction is plotted in Fig. 15, where the resulting data have been fitted to a polynomial of order four around $y_{0}: \Delta B_{z, 0}+\Delta B_{z, 010}\left(y-y_{0}\right)+\Delta B_{z, 020}\left(y-y_{0}\right)^{2}$ $+\Delta B_{z, 030}\left(y-y_{0}\right)^{3}+\Delta B_{z, 040}\left(y-y_{0}\right)^{4}$. a)

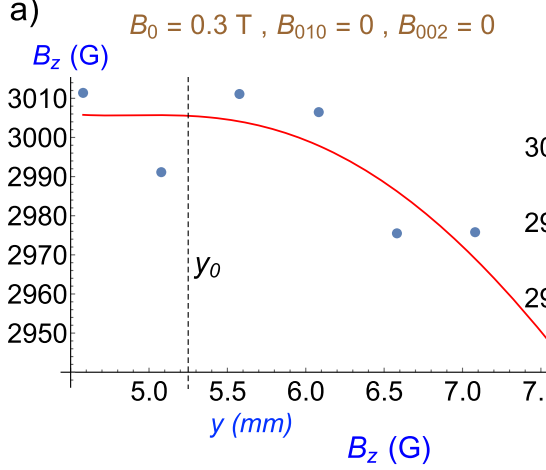

b) $B_{0}=0.3 \mathrm{~T}, B_{010}=0, B_{002}=0$

$B_{z}(\mathrm{G})$

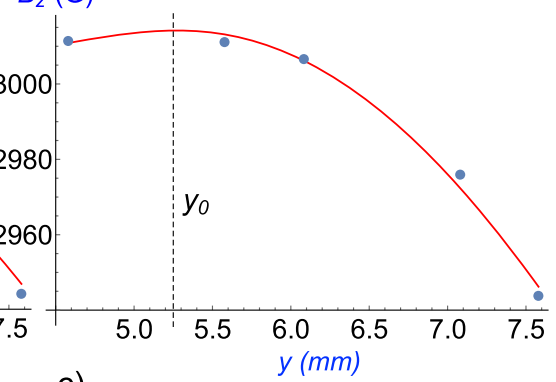

c)

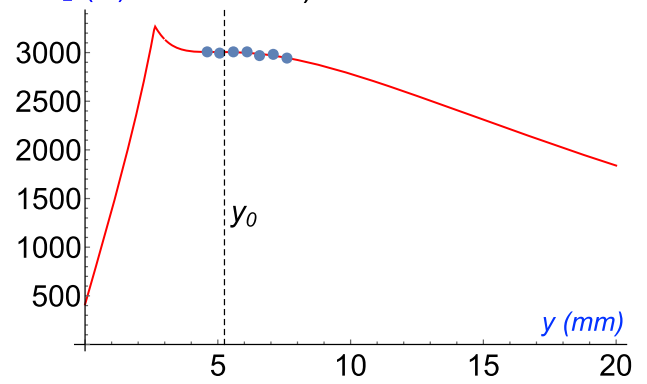

FIG. 14. (a) Result magnetic field at $y_{0}$ $=1.45 \mathrm{~mm}$ (above the geonium chip's surface) calibrated with $\Gamma^{\prime}$ of Eq. (14). The target $\tilde{B}_{\text {reduced }}$ is shown on top of the graph. Function fitted with all seven sensors. (b) Result when the Hall-sensors 2 and 5 are removed from the fit. (c) Plot of the fitted function in a bigger range of heights. In all graphs, the variable $y$ is given with the origin in the center of the magnetic field source $\left[\equiv y^{\prime}\right.$ in Fig. 8(c)]. 
Subtracted datasets

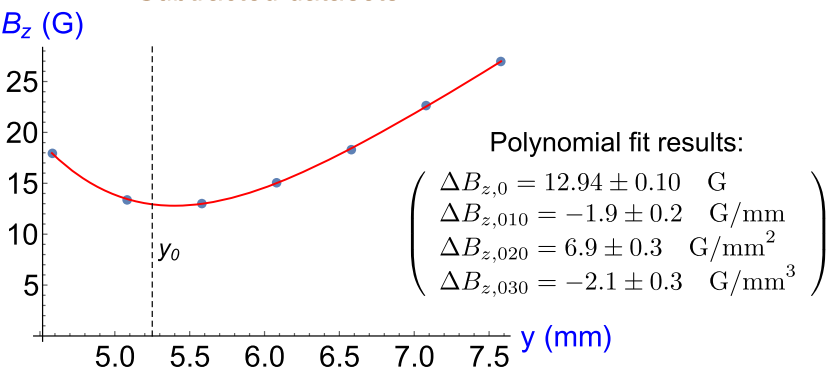

FIG. 15. Experimental data sets subtracted. The continuous curve is a fit to a polynomial of order 4 around $y_{0}$.

Figure 15 provides significant insight into the results presented in Eqs. (17) and (18). The first observation is that when subtracted, the scattering of the data around the fitted curve-clearly visible in both figures [Figs. 13(a) and 14(a)] -is greatly reduced. The fitted curve of Fig. 15 shows that all experimental data smoothly follow the fitted polynomial. The latter has the simple shape of a magnetic bottle along $\hat{u}_{y}$, which is the expected shape for Fig. 14. The perceptible reduction of the data scattering strongly suggests that at high fields, sensors 2 and 5 are subject to some offset with respect to the others, as mentioned in Sec. IV E. This is the reason for performing the analysis of Figs. 13(b) and 14(b), where the data of those two sensors have been discarded.

The second information gained from the polynomial fit of Fig. 15 is that the acquired difference coefficients, $\Delta B_{z, 0}, \Delta B_{z, 010}$, $\Delta B_{z, 020}, \Delta B_{z, 030}$, fully agree with the results obtained from subtracting the values of Eq. (17) minus the values of Eq. (18). Specifically, the polynomial fit of Fig. 15 delivers the same value for the gradient of Eq. (17) and the same curvature (with opposite sign) of Eq. (18). The key consideration here is that $\Delta B_{z, 010}$ and $\Delta B_{z, 020}$ have been obtained by fitting to a "neutral" polynomial, while the values of Eqs. (17) and (18) have been determined by fitting the current density of the theoretical curves of Eq. (12). Hence, the coincidence of both results strongly supports the procedure for measuring $\Gamma, \Gamma^{\prime}$ and the residual inhomogeneities discussed in Secs. IV B and IV E, respectively. The use of the theoretical functions of Eq. (12) does not bias the results obtained.

It might be thought that instead of using Eq. (12), the measurement of $\Gamma$ and residual inhomogeneities could have been done with simple polynomial fits, as in Fig. 15. However, when fitting the datasets of Fig. 10 with a polynomial, the results are significantly less accurate than when using Eq. (12). In the latter case, only one or two parameters are fitted (current density and "tilt angle"), while in the former case, at least four parameters- $B_{z, 0}, B_{z, 010}, B_{z, 020}$, and $B_{z, 030}-$ must be fitted, and this with only seven data points available. When using polynomial fits with 4 degrees of freedom, the scattering of the data due to the relative offsets discussed above turns into imprecise values for $B_{z, 020}$ and very unreliable for $B_{z, 030}$. As a result, the so obtained $\Gamma$ matrix is inevitably less accurate. An example is plotted in Fig. 16, showing a target magnetic field $\tilde{B}_{\text {reduced }}=\left(B_{z, 0}=0.5 \mathrm{~T}\right.$, $\left.B_{z, 010}=0, B_{z, 020}=0\right)$. In this case, the $\Gamma$ matrix and, therefore, the used currents have been determined by polynomial fitting of the calibration data of Fig. 10. The measured residual inhomogeneities

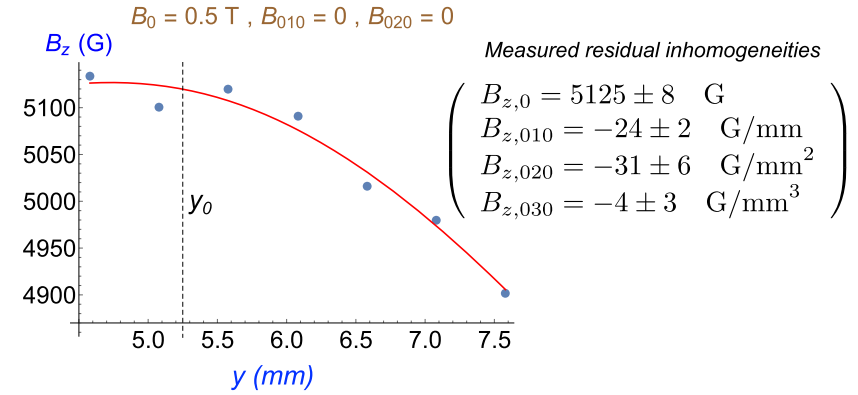

FIG. 16. Example of magnetic field obtained by calibration of the source using polynomial fitting of the experimental data.

in Fig. 16 have been obtained with the same method as in Sec. IV E. Clearly, the residual gradient and curvature in that example are much higher than those of the examples of Figs. 13 and 14.

\section{SUMMARY AND OUTLOOK}

The examples of compensated fields presented in Figs. 13 and 14 demonstrate the ability of the magnetic field source to provide a magnetic field tailored to the needs of a cryogenic ion trap with a single electron/ion. The residual inhomogeneities reported in Eq. (18) show the ability to eliminate the gradient, and Eq. (17) shows the ability to eliminate the curvature. This latter example has been chosen with a very small but not vanishing gradient in order to check the validity of our approach for measuring the residual inhomogeneities, as discussed in Sec. IV F. Many more examples have been measured among others showing the capability of the source to address the full set $\tilde{B}$, hence explicitly eliminating $B_{z, 030}$ too. ${ }^{33}$ The magnetic field reported in Eq. (18) is a gradient-free magnetic bottle, with a curvature about six times smaller than the one used for measuring the $g$-factor of the free electron ${ }^{35}$ and about 20 times smaller than that in the first observation of the continuous Stern-Gerlach effect with a highly charged ion. ${ }^{36}$ Both these are prominent examples of the great accuracy of Penning trap technology, and our source provides a field of similar or even better spatial homogeneity. As discussed in Sec. II, the amplitude of the motion of a trapped electron/ion amounts to $\sim 100 \mu \mathrm{m}$. The measurements of Figs. 13 and 14 show the consistency of the magnetic field within a region $y_{0} \pm 0.5 \mathrm{~mm}$, hence fully sufficient for a cryogenic ion trap.

It must be strongly emphasized that the fields reported in Eqs. (17) and (18) do not represent the limit of the source, rather they only represent the limit of the calibration procedure. The small amount of sensors and their spacing of $0.5 \mathrm{~mm}$ cap the accuracy of the calibration discussed in Sec. IVC. However, the measured fields and the currents reported in Eq. (16) are only the starting point from which the trapped ion/electron itself can further act as a magnetic sensor, using the Gabrielse-Brown invariance theorem. ${ }^{37}$ This will then allow us to substantially improve the calibration and reduce even more the residual gradient and curvature. While in its current basic version, only $\tilde{B}$ with three or four variables can be addressed, adding extra currents will enable eliminating additional higher-order inhomogeneities.

We have demonstrated fields of up to $0.5 \mathrm{~T}$ with our basic planar magnetic field source. A robust simulation has been used to 
analyze the data measured at $4 \mathrm{~K}$ and provide evidence that our prototype is performing as expected and is well matched to the theory functions. The field strength is limited by the amount of current density achievable within the wires. The source is close to the critical current of the $\mathrm{NbTi}$ wire used. Although thinner diameter wires are commercially available, the $100 \mu \mathrm{m}$ wires are approaching the limit of what is practical in terms of physically winding coils, as they can be easily damaged. We are currently developing the next generation of this magnetic field source, ${ }^{33}$ made not with wires but precisely machined from a solid block of superconducting material and which will run with persistent currents, without the need of external current supplies. For this, we have developed a specially designed flux pumping technique, which allows magnetizing the solid superconducting block to potentially hundreds or thousands of amperes from very small input, seed currents. ${ }^{12}$ This promises to open the way toward a planar magnetic field source with much stronger, above $1 \mathrm{~T}$, homogeneous fields.

\section{ACKNOWLEDGMENTS}

This work was supported by the UKRI-EPSRC, through Grant Nos. EP/N003675/1 and EP/R008558/1.

\section{DATA AVAILABILITY}

The data that support the findings of this study are available within the article and the cited documents.

\section{REFERENCES}

${ }^{1}$ M. Stehling, R. Turner, and P. Mansfield, Science 254, 43 (1991).

${ }^{2}$ L. M. K. Vandersypen and I. L. Chuang, Rev. Mod. Phys. 76, 1037 (2005).

${ }^{3}$ A. G. Marshall and T. Chen, Int. J. Mass Spectrom. 377, 410 (2015).

${ }^{4}$ C. L. Hendrickson, J. P. Quinn, N. K. Kaiser, D. F. Smith, G. T. Blakney, T. Chen, A. G. Marshall, C. R. Weisbrod, and S. C. Beu, J. Am. Soc. Mass Spectrom. 26, 1626 (2015).

${ }^{5}$ A. Marshall, Fourier Transform Mass Spectrometry, Spectroscopy in the Biomedical Sciences (CRC Press, 2018).

${ }^{6}$ K. Blaum, H. Kracke, S. Kreim, A. Mooser, C. Mrozik, W. Quint, C. C. Rodegheri, B. Schabinger, S. Sturm, S. Ulmer, A. Wagner, J. Walz, and G. Werth, J. Phys. B: At., Mol. Opt. Phys. 42, 154021 (2009).

${ }^{7}$ R. S. Van Dyck, Jr., D. L. Farnham, S. L. Zafonte, and P. B. Schwinberg, Rev. Sci. Instrum. 70, 1665 (1999).

${ }^{8}$ G. Gabrielse and J. Tan, J. Appl. Phys. 63, 5143 (1988).
${ }^{9}$ Y. Iwasa, J. Bascuan, S. Hahn, M. Tomita, and W. Yao, IEEE Trans. Appl. Supercond. 20, 718 (2010).

${ }^{10}$ C. Hoffmann, D. Pooke, and A. D. Caplin, IEEE Trans. Appl. Supercond. 21, 1628 (2011).

${ }^{11}$ L. J. M. van de Klundert and H. H. J. ten Kate, Cryogenics 21, 195 (1981).

${ }^{12}$ J. H. Lacy, A. Cridland, J. Pinder, A. Uribe, R. Willetts, and J. Verdú, IEEE Trans. Appl. Supercond. 30, 1 (2020).

${ }^{13}$ A. Cridland, J. H. Lacy, J. Pinder, and J. Verdú, Photonics 3, 59 (2016).

${ }^{14}$ F. Crimin, B. M. Garraway, and J. Verdú, J. Mod. Opt. 65, 427 (2018).

${ }^{15}$ J. Verdú, New J. Phys. 13, 113029 (2011).

${ }^{16}$ J. Pinder and J. Verdú, Int. J. Mass Spectrom. 356, 49 (2013).

${ }^{17}$ J. Pinder, "The geonium chip: Engineering a scalable planar penning trap," Ph.D. thesis, University of Sussex, Falmer, UK, 2017.

${ }^{18}$ F. Xian, C. L. Hendrickson, and A. G. Marshall, Anal. Chem. 84, 708 (2012).

${ }^{19}$ J. Kelley, Applications of Cryogenic Technology (Plenum Press, New York and London, 1991), Vol. 10.

${ }^{20}$ D. Barron, M. Atlas, B. Keating, R. Quillin, N. Stebor, and B. Wilson, Cryocoolers 17, 555 (2012).

${ }^{21}$ S. Stahl, F. Galve, J. Alonso, S. Djekic, W. Quint, T. Valenzuela, J. Verdú, M. Vogel, and G. Werth, Eur. Phys. J. D 32, 139 (2005).

${ }^{22}$ J. Verdú, “Ion trap,” U.S. Patent 8362423 B1 (29 January 2013).

${ }^{23}$ J. Verdú, "Ion trap," WO Patent 2013/041615 A2 (28 March 2013).

${ }^{24}$ H. G. Dehmelt, Proc. Natl. Acad. Sci. U. S. A. 83, 2291 (1986).

${ }^{25}$ J. Verdú, S. Djekic, S. Stahl, T. Valenzuela, M. Vogel, G. Werth, T. Beier, H.-J. Kluge, and W. Quint, Phys. Rev. Lett. 92, 093002 (2004).

${ }^{\mathbf{2 6}}$ See www.supercon-wire.com/content/nbti-superconducting-wires for Supercon, Inc., 2015.

${ }^{27}$ A. Al-Rjoub and J. Verdú, Appl. Phys. B 107, 955 (2012).

${ }^{28}$ S. Ulmer, K. Blaum, H. Kracke, A. Mooser, W. Quint, C. C. Rodegheri, and J. Walz, Nucl. Instrum. Methods Phys. Res., Sect. A 705, 55 (2013).

${ }^{29}$ J. Ekin, Experimental Techniques for Low-Temperature Measurements: Cryostat Design, Material Properties and Superconductor Critical-Current Testing (Oxford University Press, 2006).

${ }^{30}$ A. Cridland, "Development of a planar penning trap for quantum applications with electrons," Ph.D. thesis, University of Sussex, Falmer, UK, 2018.

${ }^{31}$ See http://www.superpower-inc.com/content/2g-hts-wire for Superpower, Inc.

${ }^{32}$ See http://www.arepoc.sk/?p=home for Arepoc.

${ }^{33}$ J. H. Lacy, "Development of a planar magnetic field source for the geonium chip Ppnning trap," Ph.D. thesis, University of Sussex, Falmer, UK, 2019.

${ }^{34}$ See www.prototools.co.uk/high-z-t-400-cnc-machine.html for Prototools, 2016. ${ }^{35}$ D. Hanneke, S. Fogwell, and G. Gabrielse, Phys. Rev. Lett. 100, 120801 (2008).

${ }^{36}$ N. Hermanspahn, H. Häffner, H.-J. Kluge, W. Quint, S. Stahl, J. Verdú, and G. Werth, Phys. Rev. Lett. 84, 427 (2000).

${ }^{37}$ G. Gabrielse, Int. J. Mass Spectrom. 279, 107 (2009). 